\title{
Análise da produção dos congressos brasileiros de comunicação suplementar e/ou alternativa: tendências e perspectivas
}

\section{Review of the production of brazilian conferences of augmenta- tive and alternative communication: trends and prospects}

\begin{abstract}
Resumo: A Comunicação Suplementar e/ou Alternativa (CSA) encontra-se em expansão no Brasil, sendo ainda, pouco documentada. Este trabalho trata-se da análise documental dos anais e livros dos quatro primeiros congressos brasileiros de CSA, com o objetivo de mapear e conhecer a produção científica para levantar e identificar perspectivas e lacunas. Foram analisados 323 trabalhos, nas categorias: área do trabalho, tipo de instituição, população-alvo e ciclos de vida dos usuários. Os resultados mostram maior variedade nas categorias ao longo dos anos, evidenciando tendência de ampliação da CSA além da Saúde e Educação, abrangendo as áreas de Arte e Tecnologia; diversos ciclos de vida; outros interlocutores, como educadores e graduandos e outros ambientes além do aumento entre as parcerias de Centros especializados e Universidades. As mudanças abrem perspectivas de um novo olhar da CSA no país, abrangendo questões de funcionalidade, participação e inclusão em consonância com as políticas públicas de Saúde e Educação.

Palavras-chave: Comunicação Suplementar e Alternativa. Revisão sistemática. Classificação Internacional de Funcionalidade, Incapacidade e Saúde.
\end{abstract}

Abstract: The Augmentative and Alternative Communication (AAC) is increasing in Brazil, but it is still little documented. This work consists in the documentary analysis of Annals and books of the first four Brazilian AAC conferences, with the objective of mapping the scientific production to raise and to identify prospects and gaps. We analyzed 323 studies in the following categories: work field, type of institution, target population and life cycles of AAC users. The results show greater variety in the categories over the years, which demonstrate an increasing trend in AAC in addition to Health and Education, comprising Art and Technology; several life cycles; other interlocutors (educators and undergraduate students) and environments besides an increase in partnerships between specialized centers and universities. The changes open up prospects for a new look at the AAC in Brazil covering issues of functionality, participation and inclusion in line with public policies in Health and Education.

Keywords: Augmentative and Alternative Communication. Review. International Classification of Functioning. Disability and Health.

CHUN Regina Yu Shon; MOREIRA, Eliana Cristina; SANTANA, Maria Tereza Maynard; DALLAQUA Graziella Batista. Análise da produção dos congressos brasileiros de comunicação suplementar e/ou alternativa: tendências e perspectivas. Informática na Educação: teoria e prática, Porto Alegre, v. 16, n. 2, p. 63-76, jul./dez. 2013.

\author{
Regina Yu Shon Chun \\ Eliana Cristina Moreira \\ Maria Tereza Maynard Santana \\ Graziella Batista Dallaqua \\ Universidade Estadual de Campinas
}

\section{Introdução}

O momento político atual de diálogo, pactuação de políticas e de um ambiente propício à efetivação dos direitos das pessoas com deficiência exige um trabalho urgente de aprofundamento em relação às questões sociais, econômicas e políticas, para sustentar a capacidade de intervir e propor soluções efetivas de inclusão nos mais diversos campos da realização da cidadania desta expressiva parcela do povo brasileiro ( $A$ Convenção sobre Direitos das Pessoas com Deficiência Comentada. CORDE, 2008, p. 18).

A Convenção sobre Direitos das Pessoas com Deficiência realizada em 2006, adotada pela Organização das Nações Unidas e ratificada pelo Brasil (CORDE, 2008), cujo trecho intro- 
duz este artigo, pode ser considerada um marco em defesa dos direitos humanos e de vida desse grupo populacional por envolvê-las no processo de construção desse documento junto com os governos dos países membros da ONU (DUDAS, 2013), assumindo assim, "papel de ser um indutor das transformações sociais visando caminhar para uma sociedade inclusiva" (CORDE, 2008, p. 18). Trata-se de documento que apresenta os direitos humanos, avanços e conquistas das pessoas com deficiência no Brasil em sua própria ótica (CORDE, 2008).

Como colocado no Relatório Mundial sobre a Deficiência, publicado pela Organização Mundial de Saúde (OMS) em 2011, pessoas com deficiência exigem diferentes tipos de intervenções relativamente menores e baratas ou complexas e caras. Dentre as quais, situa-se a Comunicação Suplementar e/ou Alternativa (CSA). Além disso, como pontua Dudas (2013, p. 86): "Com a Convenção da ONU, se não houver acessibilidade, significa que há discriminação, condenável do ponto de vista moral, ético e punível na forma da lei".

Diante da importância do tema e tendo em vista contribuir com essa discussão, busca-se analisar os quatro primeiros Congressos de CSA do Brasil, realizados de 2005 a 2011, em continuidade a estudo anterior (CHUN et al., 2012), com o objetivo mapear a produção científica e verificar tendências e perspectivas da CSA em nosso país a partir desses eventos. Ressalva-se que este trabalho representa uma leitura das autoras em relação a esses dados, revendo-se o posicionamento em publicação anterior (CHUN et al., 2012) quanto ao que se considerou como "estado da arte".

Segundo Ferreira (2002), nas últimas décadas verifica-se um desenvolvimento significativo de estudos denominados de "estado da arte" ou "estado do conhecimento", de caráter bibliográfico com o desafio comum de mapear e de discutir a produção acadêmica em diferentes campos do conhecimento em função da carência de conhecimento em determinada área, como no caso da temática em questão.

A autora pontua dificuldades e limitações desse tipo de investigação, particularmente, em relação aos resumos de dissertações, questionando se estes possibilitam análise dos denominados estudos de "estado da arte", cujos dados pelo tipo de informação pertinente, dependerão da interpretação do pesquisador e, portanto, segundo a autora, a história da produção analisada representa aquilo que o pesquisador lê/interpreta.

Apesar do crescimento da CSA no Brasil, encontram-se ainda, poucos estudos de revisão (MANZINI, 2007; BERBERIAN et al., 2009; CHUN, 2009; MANZINI, 2011, CHUN et al. 2012 ), que mostram a carência nesse aspecto e reiteram a importância de trabalhos como aqui proposto, em que pesem as ressalvas desse tipo de análise, discutidas por Ferreira (op. cit.).

A CSA em nosso país vem se ampliando, desde os trabalhos pioneiros desenvolvidos no final dos anos 70 em clínicas e centros especializados (REILY, 2007; CHUN et al., 2012), em uma combinação com escolas especiais (NUNES, 2007). Verifica-se maior porcentagem de trabalhos em prefeituras de diversas regiões do país, principalmente por intermédio das Secretarias Municipais de Saúde e de Educação, visando favorecer a comunicação e a inclusão dos alunos com necessidades educacionais especiais (REILY, 2007; CHUN et al., 2012). Além disso, observa-se importante crescimento nos círculos acadêmicos (NUNES, 2007; REILY, 2007; CHUN et al., 2012).

Os avanços técnico-científicos na Saúde e na Educação têm possibilitado maior saúde 
e qualidade de vida à população como também gerado crescente aumento das demandas daqueles que necessitam formas alternativas de comunicação, como a CSA pode proporcionar (CHUN, 2009; CHUN et al., 2012). A CSA constitui importante ferramenta para que essas pessoas possam expressar desejos e sentimentos e conquistar maior independência e autonomia por meio de diferentes recursos de tecnologia assistiva.

A CSA trata-se de abordagem clínico-educacional que visa, de forma temporária ou permanente, o apoio, a complementação, a suplementação ou a substituição das formas de produção e interpretação verbal (CHUN, 2009; CHUN et al., 2012). Trata-se de área que avançou significativamente em sua prática em uma abordagem multi e interdisciplinar a partir da utilização de recursos de baixa e alta tecnologia.

Nos quarenta anos de história da CSA, as práticas se transformaram. Light e Mcnaughton (2012) pontuam o avanço da CSA nas últimas décadas, destacando as grandes mudanças no cenário internacional que, como ressaltam os autores, refletem os desafios futuros para favorecer a comunicação e a qualidade de vida das pessoas que necessitam da CSA bem como de seus familiares.

Zangari et al. (1994), em clássica revisão da CSA, no periódico Augmentative and Alternative Communication, publicação especializada da área, pontuam que, apesar da história ser construída a cada dia, somente poderá ser lembrada e avaliada se houver registros escritos disponíveis. Os autores acrescentam que muito se fez nesse curto período da CSA, no mundo, porém, muitas pessoas ainda desconhecem esse trabalho e sua evolução. Situação que persiste nos dias atuais. Como aborda esse artigo, dentre as dificuldades para se tra- çar um panorama histórico da CSA no cenário internacional, encontram-se trabalhos não documentados, realidade comum também em nosso país.

De modo que o mapeamento dos quatro primeiros congressos de CSA do Brasil, foco deste trabalho, assume grande relevância, especialmente na fase atual de desenvolvimento, ou seja, de crescente expansão, para que se possa conhecer a produção da área, levantar e identificar perspectivas, lacunas e vieses, como pontua Soares (1987 apud FERREIRA, 2002), além de se considerar carência de registros e publicações na área.

Os achados poderão demonstrar tendências da produção da CSA nos primeiros congressos brasileiros de comunicação alternativa e apontar caminhos que possam ser mais explorados, em consonância com as políticas atuais de saúde e educação tendo em vista a participação, funcionalidade e inclusão das pessoas com deficiências.

\section{Referencial teórico}

No atual cenário nacional observa-se crescente expansão da CSA, mas é fato que essa área ainda, apresenta ainda um reduzido número de publicações, aspecto em crescimento, como mostram alguns estudos (BERBERIAN et al. 2009; CHUN, 2009; MANZINI, 2009, 2011).

Berberian et al. (2009) analisaram periódicos indexados na área de fonoaudiologia até 2005, computando 11 publicações, resultado que evidencia a produção restrita em CSA e a necessidade de ampliação de trabalhos em outras áreas para se poder traçar um panorama mais abrangente desse campo.

Chun (2009) revisou os artigos nas bases de dados LILACS, SCIELO e MEDLINE para dis- 
cutir as versões brasileiras referentes à "Augmentative and Alternative Communication" e registrou 16 trabalhos. Diversos são os pontos necessários para aprofundamento da discussão sobre a terminologia, sendo que, dentre estes, a autora também abordou em suas conclusões, o baixo número de publicações.

Manzini $(2009,2011)$ realizou mapeamento das pesquisas em CSA na Pós-graduação brasileira. Segundo esse pesquisador, "a análise de temas de pesquisa não pode ser considerada como um simples levantamento bibliográfico. Ela guarda relevância no meio acadêmico, pois serve como indicador de políticas para fomentar pesquisas em áreas carentes e prioritárias" (MANZINI, 2009, op. cit., p. 312). Para tanto "é necessário que a análise temática seja exaustiva e sistemática" (op. cit., p. 312). Em 2011, Manzini se debruça novamente sobre o Banco de Teses da Capes em continuidade à pesquisa e complementando os dados, analisa a formação dos orientadores e orientandos. Dentre suas conclusões, o autor pontuando dois indicativos nesse panorama, quais sejam, o aumento de número de orientadores e a continuidade dos orientandos no trabalho com Comunicação Suplementar e/ou Alternativa, como importantes alavancas no crescimento das pesquisas na área.

No cenário internacional, Light e McNaughton (2012) apontam uma significativa mudança na participação social dos sujeitos que usam a CSA, o que tem levado a maior reflexão e ampliação dos estudos, além de maior participação de outras áreas de atuação. Segundo os autores, dados demográficos internacionais mostram significativo aumento da população usuária de CSA, com evidência de seus benefícios e da ampliação de serviços e profissionais que trabalham com a CSA. Considerando-se que não existem dados oficiais brasileiros, estudos como aqui proposto, podem contribuir para um mapeamento do cenário da CSA em nosso país, a partir da análise da produção dos congressos nacionais.

As colocações até aqui expostas reafirmam a relevância de se dar continuidade à análise dos três primeiros congressos brasileiros de CSA (CHUN et al., 2012), cujos resultados permitiram vislumbrar caminhos a serem percorridos no atual estudo, abrangendo-se análise de aspectos tais como as áreas do trabalho, além da Saúde e Educação, Tecnologia e Artes; o tipo de instituição e a população alvo, buscando-se analisá-los sob uma abordagem de funcionalidade e participação dos interlocutores e do próprio usuário no processo.

No campo da Saúde, os avanços tecnológicos têm possibilitado maior sobrevida das pessoas de diferentes faixas etárias, sendo que em casos com alterações ou disfunções neurológicas, há consequente aumento da demanda de assistência à saúde e à educação desse grupo populacional, os quais, dentre outros aspectos, necessitam de formas alternativas para se comunicar como a CSA pode propiciar (CHUN, 2009, CHUN et al. 2012).

A singularidade das práticas de implementação da CSA em relação ao design, relevância e velocidade do processamento de informação além do trabalho com o uso da CSA nos diversos contextos sociais e com diferentes parceiros são fundamentais para uma boa intervenção, generalização e manutenção do uso da CSA (CESA et al., 2010).

Segundo Manzini e Deliberato (2006), em material publicado pela Secretaria de Educação Especial do Ministério da Educação (MEC), colocam que na Educação Especial, os recursos da CSA são um meio eficaz para garantir a inclusão de alunos com necessidades especiais na área da comunicação no processo de inte- 
ração entre professor e aluno, de modo que o educando possa se "...comunicar com as outras pessoas e expor suas idéias, pensamentos e sentimentos se puder utilizar recursos especialmente desenvolvidos e adaptados para o meio no qual está inserido" (op. cit., p. 4).

Além disso, dentre as políticas estabelecidas pelo MEC para escolas inclusivas, recomenda-se "propiciar melhores níveis de interação e comunicação" e "adotar sistemas de comunicação suplementares e/ou alternativos" (BRASIL, 2005).

Outro aspecto em pauta, na prática da CSA no Brasil diz respeito ao uso de recursos de baixa e alta tecnologia. As últimas duas décadas evidenciam grande evolução digital, possibilitando rápida transmissão das informações e aumento do poder de trocas de informações entre as pessoas. A disponibilidade de novas tecnologias cresce, sendo que a cada dia novos recursos surgem com novas funções e novos formatos (PELOSI, 2013).

No Brasil, o acesso a recursos de alta tecnologia é bastante restrito para as pessoas com deficiências, sendo necessário quebrar barreiras, como aborda Pelosi (op. cit.) tais como o fornecimento de equipamentos pelo SUS, a falta de conhecimento dos profissionais de Saúde e na Educação para indicação dos mesmos e a pequena oferta de recursos oferecidos pela indústria brasileira.

Outro ponto relevante para a discussão da temática remete às observações da American Speech-Language-Hearing Association - ASHA (2004 apud PANHAN 2011, p. 17) de que:

[...] clínicos e pesquisadores precisam considerar a Comunicação Suplementar e Alternativa (CSA) em um contexto social, em que o papel principal seja melhorar os níveis de participação ativa em eventos relevantes e interessantes para o sujeito, levando em con- ta a amplitude de ações e os componentes multifatoriais envolvidos na CSA.

Tal perspectiva mostra-se consoante com as colocações da Organização Mundial de Saúde (OMS, 2011) que recomenda uma "abordagem para dificuldades funcionais" ao invés de uma "abordagem para deficiência" para determinar sua prevalência e captar melhor a extensão da deficiência, os fundamentos da Classificação Internacional de Funcionalidade, Incapacidade e Saúde (CIF). De modo que a CIF se mostra como importante subsídio de análise para o tipo de estudo que aqui se coloca.

A CIF foi aprovada pela OMS em 2001 e retrata um modelo de funcionalidade em um sistema de classificação, com uma abordagem biopsicossocial para as dimensões de saúde abordadas. A CIF nasceu da necessidade de cobrir questões que não eram alcançadas pela CID (Classificação Internacional de Doenças), uma vez que esta fornece informações relativas ao diagnóstico das doenças, mas não em outros aspectos, como, por exemplo, as conseqüências de uma determinada doença para um determinado sujeito como é proposto pela CIF (Di NUBILA; BUCHALLA, 2008), e em especifi$\mathrm{Co}$, a dificuldade de comunicação e de participação em atividades que envolvam a comunicação, fatores estes que podem ser avaliados e trabalhados através da introdução da CSA.

A OMS, por meio da CIF, considera essenciais, aspectos como atividade, participação e fatores contextual, ambiental e pessoal. Tal ótica propicia uma visão ampliada de saúde, em que se olha para a funcionalidade do indivíduo e não apenas, para suas incapacidades (CCOMS, 2003). Este modelo influencia na inter-relação de diferentes fatores, considerando além das questões patológicas, a funcionalidade do sujeito articulada às condições físicas e 
sociais, aos mais variados contextos sociais e ao acesso aos serviços (ARAújO, 2011).

No Relatório Mundial sobre a Deficiência (OMS, 2011) destaca-se o uso da CIF como um marco universal para a coleta de dados relativos às deficiências e a elaboração de políticas públicas que visem a promoção da participação e inclusão social e saúde dos sujeitos com deficiência, considerando-se o que a CIF oferece pode-se assegurar que as diferentes fontes de dados tenham uma harmonia a partir dos resultados.

De acordo com esse documento, ainda, observa-se que algumas práticas de reabilitação têm se beneficiado de estudos, ressalvando-se, porém, que pesquisas validadas em intervenções específicas e programas de reabilitação ainda são poucas. Assinala-se a carência de estudos randomizados controlados e reconhecidos para maior eficácia na avaliação, construção de intervenções e de programas e políticas de reabilitação. Para tanto, ressalta-se que são necessárias pesquisas abrangendo diferentes contextos, acessibilidade e resultados das ações, sendo que dentre esses estudos, pode-se inserir pesquisas no âmbito da CSA.

Tendo em vista que a participação e funcionalidade é um ponto crucial neste novo olhar para as pessoas com deficiência, e que estas variáveis são relacionadas com os fatores pessoais, ambientais e com as condições de saúde de cada indivíduo, torna-se necessário melhorar a qualidade dos dados obtidos pelas pesquisas acerca da oferta de serviços, resultados e benefícios para se ter melhor planejamento e eficácia nas intervenções, além de se proporcionar maior tomada de decisões pelas pessoas com deficiência (OMS, 2011).

Neste contexto, Panhan e Monteiro (2009) destacam que a CIF constitui-se como um ins- trumento valioso, pois permite uma coleta de dados com olhar voltado para a funcionalidade e com linguagem padronizada internacionalmente, possibilitando, no caso de sua aplicação à CSA, identificar necessidades funcionais e de reabilitação dos usuários de CSA, levando-se em conta fatores físicos e sociais. Os autores pontuam que os profissionais de CSA devem se inserir nestes novos paradigmas.

Segundo Raghavendra et al. (2007), um dos principais elementos da CSA é a definição das exigências que vão ao encontro das necessidades dos usuários, de acordo com suas habilidades. Dessa forma, os aspectos como função do corpo, estrutura e atividade interagem com os sistemas de CSA, além da influência dos fatores ambientais (suporte de serviços a partir do ambiente, das atitudes e dos parceiros de comunicação) e fatores pessoais (aceitação pelo uso da CSA), considerando práticas terapêuticas de CSA a partir da singularidade dos sujeitos.

Os aspectos históricos possibilitam maior compreensão do que está sendo produzido em CSA em que pesem as restrições apontadas por Ferreira (2002), em estudos bibliográficos, especialmente no tocante aos resumos, no caso de Congressos. Em busca de soluções para essa problemática, a autora aponta "levar em consideração a natureza do material que temos em mãos a partir da noção de gênero do discurso, conforme Bakhtin (1997) e da noção do suporte material em que cada resumo se apresenta, de acordo com Chartier $(1990,1996) "$ (FERREIRA, 2002, p. 267).

Portanto, como ressalva essa autora, apresenta-se aqui uma das possíveis histórias e leituras da produção dos Congressos da CSA a partir do olhar e experiência das autoras como pesquisadoras e profissionais atuantes na CSA no Brasil. 


\section{Objetivo}

Mapear a produção (anais e livros) de Comunicação Suplementar e/ou Alternativa a partir dos quatro Congressos Brasileiros de Comunicação Suplementar e/ou Alternativa, visando levantar tendências e perspectivas.

\section{Materiais e métodos}

O estudo foi desenvolvido no Centro de Estudos, Pesquisa e Reabilitação Prof. Dr. Gabriel O. S. Porto (CEPRE) da Faculdade de Ciências Médicas da Universidade Estadual de Campinas (UNICAMP, Campinas, São Paulo). A pesquisa tem caráter de revisão, tomando como foco a produção cientifica dos Congressos Brasileiros de Comunicação Alternativa realizados em 2005, 2007, 2009 e 2011.

Para a coleta de dados, foram utilizados os Anais dos Congressos, contendo resumos e/ou trabalhos completos das sessões de pôster e comunicação oral, em formato impresso e/ou digital, além de livros publicados com alguns trabalhos completos.

Foi analisado um total de 323 trabalhos. Sendo, 75 (setenta e cinco) trabalhos do I Congresso Brasileiro de Comunicação Alternativa e I Congresso Internacional de Linguagem e Comunicação da Pessoa com Deficiência (Rio de Janeiro, 2005), 72 (setenta e dois) do II Congresso Brasileiro de Comunicação Alternativa (Campinas, 2007), 100 (cem) do III Congresso Brasileiro de Comunicação Alternativa (São Paulo, 2009) e 76 (setenta e seis) do IV Congresso de Comunicação Alternativa (Rio de Janeiro, 2011).

Foram realizadas diversas leituras do material para estabelecimento das categorias de análise, conforme discriminadas a seguir: a) Área do trabalho - foram consideradas quatro grandes área, a saber saúde, educação, arte e tecnologia;

b) Tipo de instituição - foram categorizados conforme a ocorrência em: prefeitura, universidade, centro especializado, clínica, escola regular, escola especial, instituto de internos, além de trabalhos em parceria entre centro especializado/ prefeitura, centro especializado/universidade, e clínica/universidade;

c) População-alvo - compreende: usuário de CSA, educador, familiar, profissional da saúde, graduando de saúde, graduando de educação e educador/ profissional da saúde. Ainda, nesse estudo, foram levantados os ciclos de vida dos usuários de CSA, classificados em: criança, adolescente, adulto, idoso além de trabalhos voltados a mais de um ciclo, criança/adolescente e todos os ciclos de vida.

Além disso, os trabalhos que não pertenciam à categoria em análise, como no caso de revisão da literatura foram incluídos como "não se aplica". Em todos os itens analisados incluiu-se a categoria "não informada", quando não se encontrou dado correspondente no material divulgado dos Congressos ou "não especificado", quando há menção do item sem detalhamento.

Note-se que nos materiais dos congressos estudados, observa-se que não houve um padrão nas normas e categorias de submissão de trabalhos, o que resultou na apresentação dos mesmos em diferentes formatos. Além disso, em muitos casos, os trabalhos foram disponibilizados apenas no formato de resumo. Aspectos que concorreram para alta ocorrência de itens não informados ou não especificados. Ressalta-se também que foram aceitos mais de 
uma resposta nas categorias população-alvo e ciclos de vida dada a natureza das informações disponibilizadas.

\section{Resultados e discussão}

\section{a) Área do trabalho}

Quanto à área do trabalho, os achados mostram duas áreas majoritárias, Saúde e Educação, com leve predominância de trabalhos na área de Saúde, dentre essas duas, ao longo dos quatro congressos, como demonstrado no Gráfico 1. Resultados que coincidem com as origens da história da CSA em nosso país como abordado por vários autores (NUNES, 2007; REILY, 2007; CHUN et al., 2012).

O número restrito de trabalhos nas áreas de Tecnologia e Artes reflete o início do desenvolvimento e da contribuição dessas áreas na produção cientifica dos eventos analisados.

Nos dias atuais, o uso de tecnologia e a transmissão digital das informações são realidades no cotidiano da sociedade, contudo, como pontua Pelosi (2013), esse crescimento não se reflete na vida das pessoas com deficiência e necessidades de tecnologia assistiva. Esse aspecto se reflete no baixo número de trabalhos envolvendo tecnologia nos eventos analisados, resultado que evidencia a necessidade um olhar mais específico e analítico ao uso de baixa e alta tecnologia junto aos usuários de CSA em estudos futuros.

Diversas barreiras têm dificultado o acesso desses usuários a essa tecnologia tais como: "as características dos usuários; o enfoque das pessoas que dão suporte a esses usuários; o número reduzido de pesquisas na área de CAA; e as pessoas envolvidas no desenvolvimento de produtos e políticas públicas" (PELOSI, 2013, p. 372).

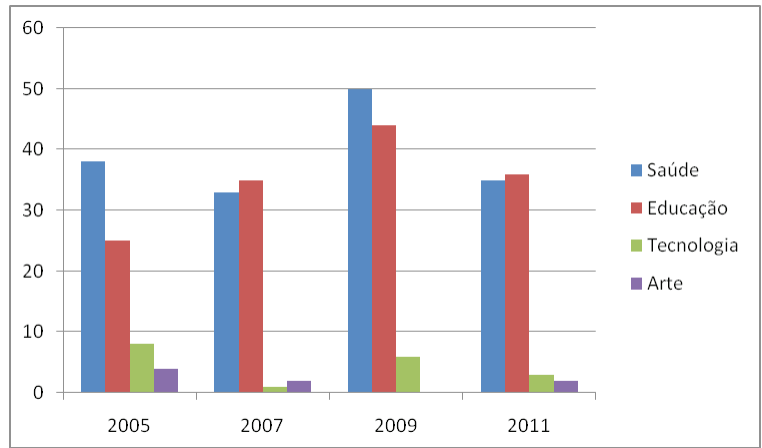

Gráfico 1. Distribuição dos trabalhos por área por ano do Congresso

\section{b) Tipo de instituição}

Segue distribuição dos trabalhos por instituição no Gráfico 2.

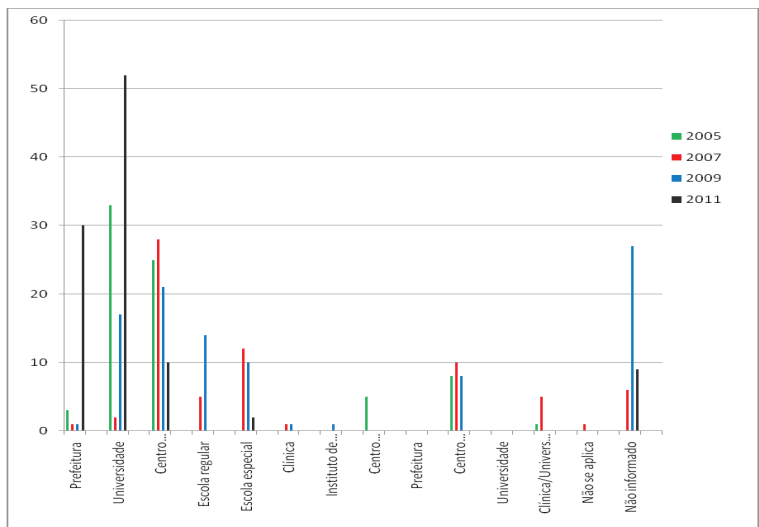

Gráfico 2. Distribuição dos trabalhos por instituição por ano de Congresso

Os achados evidenciam uma predominância de trabalhos realizados pela Universidade na porcentagem total. No entanto, note-se que em 2007 encontram-se registrados apenas dois trabalhos acadêmicos, e, em contrapartida, tal categoria representa a maior parcela em 2011. Os centros especializados são responsáveis por uma produção média similar nos 
três primeiros congressos, caindo no último evento, contudo representam a segunda categoria mais expressiva nesse item no computo total, seguidos da parceria entre Centro especializado e Universidade.

Tais resultados corroboram o crescimento nos círculos acadêmicos apontados por vários autores (NUNES, 2007; REILY, 2007; CHUN et al., 2012) bem como com a produção levantada (45 trabalhos) no mapeamento das pesquisas de CSA nos Programas de Pós-Graduação no Brasil (MANZINI, 2009), abrangendo 8 doutorados e 37 mestrados.

Verifica-se também um número significativo de trabalhos realizados por escola especial, particularmente em 2007 e 2009, assim como se observa uma parcela de trabalhos da escola regular. Tais resultados evidenciam as mudanças decorrentes do paradigma de inclusão escolar como discutido por Reily ( 2007).

\section{c) População-alvo}

A distribuição da população alvo ao longo dos Congressos são apresentados no Gráfico 3. Os resultados indicam que os usuários de CSA representam a categoria majoritária em todos os anos e no computo total. Tais achados evidenciam a preocupação com a funcionalidade e participação desse grupo populacional e reiteram a importância da CIF na CSA como abordado por alguns autores (RAGHAVENDRA et al., 2007; PANHAN; MONTEIRO, 2009).

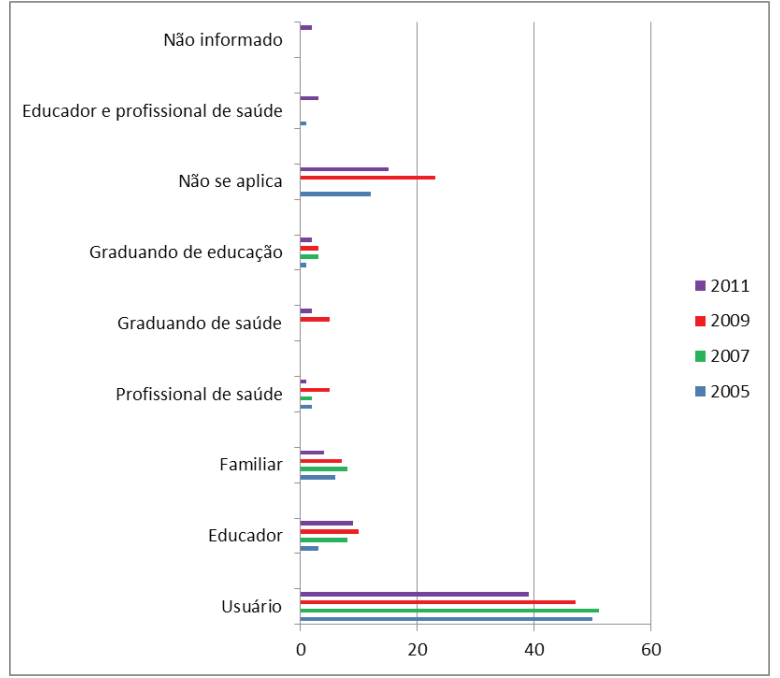

Gráfico 3. Distribuição dos trabalhos por população alvo por ano de Congresso

A CIF se revela como uma ferramenta útil para identificar e descrever as condições do usuário de CSA e o impacto na vida, possibilitando intervenções mais eficazes. Nessa mesma direção, observa-se o desenvolvimento de ações direcionadas a não-usuários de CSA, dentre eles, familiares, profissionais da saúde, da educação, dentre outros. Verifica-se assim a abrangência da CSA a outros interlocutores e contextos, que reiteram a importância dos fatores ambientais e pessoais, discutidos na CIF (CCOMS, 2003) tendo em vista um olhar integral nas ações.

Revisão realizada por Cesa et al. (2010) mostra o consenso de estudos quanto a importância da inclusão familiar para que haja um uso mais efetivo da CSA além do contexto terapêutico. Por outro lado, as autoras encontraram poucos estudos internacionais que valorizem práticas focadas na dupla relação entre usuário e família e outros parceiros conversacionais. 
Os achados evidenciam que se tem dado maior importância ao contexto e às relações sociais dos usuários de CSA e, dessa maneira, a importância em pesquisar temas que incorporem esses aspectos, entendendo um sujeito a partir da sua funcionalidade.

Outro ponto importante trazido pela CIF é a introdução dos fatores ambientais, como uma variável que pode ser uma barreira ou um facilitador na funcionalidade dos sujeitos. Desta forma, o próprio recurso de CSA é considerado um fator ambiental de comunicação e além deste recurso, os interlocutores deste sujeito, como família próxima e ampliada, amigos e colegas da comunidade onde vive, profissionais da saúde e da educação também são fatores ambientais que vão influenciar positiva ou negativamente na comunicação e participação deste sujeito na sociedade (CCOMS, 2003).

Nos últimos anos, observam-se mudanças no escopo das necessidades de comunicação, que resultou em transformações na abordagem de intervenção, com a possibilidade de acesso a comunicação via apropriação cultural e em vários ambientes (escola, casa, trabaIho), abordando desejos e necessidades, e um maior reconhecimento da amplitude de necessidades de comunicação que deve ser abordada para apoiar a participação plena na sociedade (LIGHT; MCNAUGHTON, 2012).

Segue no Gráfico 4, distribuição dos usuários de CSA quanto aos ciclos de vida.

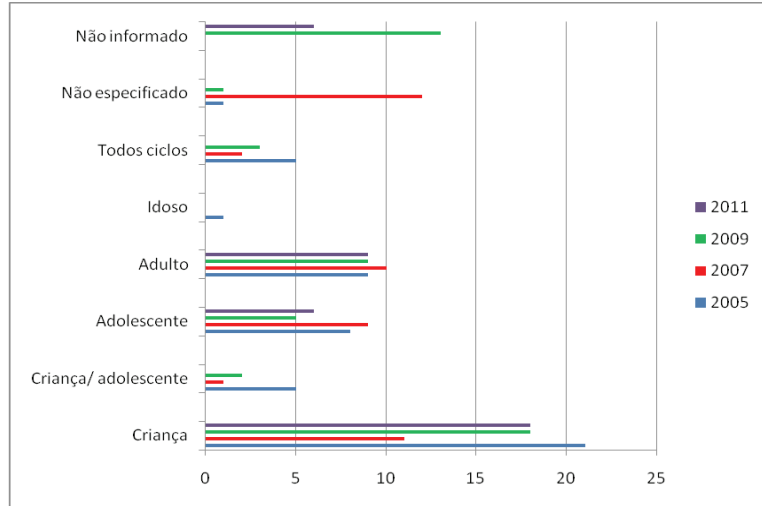

Gráfico 4. Distribuição dos usuários de CSA por ciclos de vida por Congresso

Em todos os congressos prevaleceu o trabalho voltado a crianças, mostrando que a população infantil constitui o alvo principal da intervenção com a CSA nos eventos analisados, como apontam outros autores (CESA et al., 2010; CHUN et al., 2012). Ressalva-se, porém, a ampliação do uso de CSA e o alcance das ações em outros ciclos de vida.

Os estudos envolvendo a população idosa foram minoria e indicados somente em um Congresso (2005). Os resultados mostram que poucos trabalhos abordam esse grupo populacional. Situação que merece ser revista, considerando-se que essa população encontra-se em crescimento no Brasil e no mundo, em função do aumento da expectativa de vida com o avanço no campo da saúde e da melhoria nas condições de vida nas grandes cidades de nosso país.

Observa-se uma parcela significativa em 2007, de população não especificada ou não informada, como nos anos de 2009 e 2011. 


\section{Conclusão}

Os resultados mostram maior abrangência das ações nas diversas categorias analisadas ao longo dos anos, evidenciando uma tendência de ampliação da CSA além da Saúde e Educação, envolvendo Arte e Tecnologia.

A maior parte dos estudos, ainda se encontra voltada para o usuário e a aplicação da CSA, contudo nota-se maior preocupação com outros interlocutores, tais como aqueles não usuários da CSA, como educadores e graduandos, assim como envolvendo outros ambientes além da família, tais como a escola, tanto especial quanto regular. Essas mudanças refletem uma ampliação da abordagem de participação e funcionalidade nos trabalhos, como preconizado pela CIF, que se evidencia ao longo dos congressos brasileiros de CSA, quando se volta o olhar da pesquisa para além do usuário e se abrange os "não usuários" e sua relação com as pessoas usuárias de CSA.
Além disso, apesar da predominância da atenção à população infantil, observa-se que da infância a senescência, os diferentes ciclos de vida são sujeitos das ações com a CSA no material analisado.

O tipo de instituição responsável pelos trabalhos tem se diversificado, sendo que as pesquisas estão crescendo nas Universidades ou em parcerias com elas, mudando o foco dos primórdios da CSA em nosso país, que abrangiam os centros especializados. Tal situação evidencia que a CSA está se ampliando para além da clínica e do modelo reabilitacional.

Os achados permitem uma leitura acerca do conhecimento produzido de Comunicação Suplementar e/ou Alternativa no Brasil, a partir dos Congressos de CSA, indicando uma tendência de maior diversidade de produção científica da área. As mudanças abrem perspectivas de um novo olhar da CSA no país abrangendo questões de funcionalidade, participação e inclusão em consonância com as atuais políticas públicas de Saúde e de Educação.

\section{Referências}

ARAÚJO, E. S. Manual de utilização da CIF em Saúde Funcional. Editora Andreoli: São Paulo, 2011, vol. 1, p.13-33.

BAKHTIN, M. M. Estética da criação verbal. São Paulo: Martins Fontes, 1997.

Berberian, A. P.; KRUGer, S.; GUARINello, A. C.; MASSi, G. A. A. A Produção do Conhecimento em Fonoaudiologia em Comunicação Suplementar e/ou alternativa: análise de periódicos. CEFAC. 2009; v. 11 , Supl2, p. 258-66.

BRASIL. Ministério Da Educação. Saberes e Práticas de Inclusão: recomendações para a construção de escolas inclusivas. Coordenação geral SEESP/MEC. Brasília, Secretaria da Educação Especial, 2005. 
CCOMS - Centro Colaborador da Organização Mundial da Saúde para a Família de Classificações Internacionais (Org.); Coordenação da tradução de Cassia Maria Buchalla. CIF: Classificação Internacional de Funcionalidade, Incapacidade e Saúde. São Paulo: Editora da Universidade de São Paulo, 2003.

CESA, C. C.; RAMOS-SOUZA, A. P., KASSLER, T. M. Novas perspectivas em comunicação suplementar e/ou alternativa a partir da análise de periódicos internacionais. CEFAC. 2010, v. 12 n. 5, p. 870-80.

CHUN, R. Y. S. Comunicação Suplementar e/ou Alternativa: abrangência e peculiaridades dos termos e conceitos em uso no Brasil. Pró-Fono: Revista de Atualização Científica. 2009, v. 21, n. 1, p. 69-74.

.; MOREIRA, E. C.; DALLAQUA, G. B. Estado da Arte da Comunicação Suplementar e/ou Alternativa: análise dos primeiros congressos brasileiros. Informática na Educação: Teoria \& Prática. 2012, v. 15, n. 2 , p. $199-214$.

CORDE. A Convenção sobre Direitos das Pessoas com Deficiência Comentada. Coordenação de Ana Paula Crosara Resende e Flavia Maria de Paiva Vital. Brasília: Secretaria Especial dos Direitos Humanos. Coordenadoria Nacional para Integração da Pessoa Portadora de Deficiência, 2008.

Di NUBILA, H. B. V.; BUCHALLA, C. M. O papel das Classificações da OMS - CID e CIF nas definições de deficiência e incapacidade. Rev. Bras. Epidemiol. 2008, v.11, n. 2, p. 324-35.

DUDAS, T. L. A Comunicação Alternativa como potencializadora da inclusão escolar?. In: PASSERINO, L. M.; BEZ, M. R; PEREIRA, A. C. C; PERES, A. (Orgs). Comunicar para incluir. CRBF, $1^{\text {a }}$ ed. Porto Alegre, 2013.

FERREIRA, N. S. de A. As Pesquisas Denominadas "Estado da Arte". Educação e Sociedade. 2002, v. 23, n.79, p. 257-72.

LIGHT, J.; MCNAUGHTON, D. The changing face of Augmentative and Alternative Communication: past, present and future challenges. Augmentative and Alternative Communication. 2012, v. 28, n. 4, p. 197-204.

MANZINI, E. J.; DELIBERATO, D. Portal de ajudas técnicas para a educação. Equipamento e material pedagógico especial para educação, capacitação e recreação da pessoa com deficiência física: recursos para comunicação alternativa. [2ª ed.]. Brasília: MEC/SEESP, 2006.

MANZINI, E. J. Formação continuada do professor para atender à Educação Inclusiva. Ensaios Pedagógicos: Educação Inclusiva: direito à diversidade. Brasília: 2007. 
MANZINI, E. J. Um estudo sobre as pesquisas em comunicação alternativa na pós-graduação brasileira. In: DELIBERATO, D.; GONÇALVES, M. D.; MACEDO, E. C. Comunicação Alternativa: Teoria, prática, tecnologias e pesquisa. São Paulo: Memnon Edições Científicas. 2009, p. 312-21.

MANZINI, E. J. Formação de pesquisadores para a área de comunicação alternativa. In: NUNES, L. R. O. P.; PELOSI, M. B.; WALTER, C. C. F. Compartilhando Experiências: Ampliando a Comunicação Alternativa. Rio de Janeiro: ABPEE. 2011, p. 130-48.

NUNES, L. R. d'O. de P.; NUNES, D. R. de P. Um Breve Histórico da Pesquisa em Comunicação Alternativa na UERJ. In: NUNES, L. R.; PELOSI, M. B.; GOMES, M. R. (Org.). Um Retrato da Comunicação Alternativa no Brasil: relatos de pesquisas e experiências. Rio de Janeiro: Ed. Quatro Pontos, 2007, v. 1 , p. $19-32$.

OMS. Organização Mundial da Saúde. Relatório Mundial sobre a Deficiência. 2011.

PANHAN, H. M. S.; MONTEIRO, C. B. M. Apresentação e proposta de utilização da classificação internacional de funcionalidade na comunicação suplementar e alternativa. In: DELIBERATO, D.; GONÇALVES, M. D.; MACEDO, E. C. Comunicação Alternativa: Teoria, prática, tecnologias e pesquisa. São Paulo: Memnon Edições Científicas, 2009, p. 63-72.

PANHAN, H. M. S. A Clínica com adultos e a Comunicação Alternativa: experiências compartilhadas apoiadas na CIF. In: NUNES, L. R. O. P.; PELOSI, M. B.; WALTER, C. C. F. Compartilhando Experiências: Ampliando a Comunicação Alternativa. Rio de Janeiro: ABPEE. 2011, p. 17-27.

PELOSI, M. B. Dispositivos Móveis para Comunicação Alternativa: primeiros passos. In: PASSERINO, L. M.; BEZ, M. R; PEREIRA, A. C. C; PERES, A (Orgs.). Comunicar para incluir. CRBF, $1^{\text {a }}$ ed. Porto Alegre, 2013, p. 371-80.

RAGHAVENDRA, P.; BORNMAN, J.; GRANLUND, M.; BJÖRCK-AKESSON, E. The World Health Organization's International Classification of Functioning, Disability and Health: Implications for Clinical and Research Practice in the field of Augmentative and Alternative Communication. Augmentative and Alternative Communication. 2007, v.23, n.4, p. 349-61.

REILY, L. Sobre como o Sistema Bliss de comunicação foi introduzido no Brasil, divulgado entre profissionais que trabalhavam com pessoas não falantes ou sem fala funcional, posteriormente ganhando adeptos e críticos que buscaram outras opções de sistemas pictográficas e como a informática veio a contribuir para a autonomia dos usuários de modo que eles pudessem constituir-se como cidadãos de escrita autônoma. In: NUNES, L. R.; PELOSI, M. B.; GOMES, M. R. (Org.). Um Retrato da Comunicação Alternativa no Brasil: relatos de pesquisas e experiências. Rio de Janeiro: Ed. Quatro Pontos, 2007. v. 2, p. 19-45. 
ZANGARI, C.; LOYD, L. L.; VICKER, B. Augmentative and Alternative Communication: an historic perspective. Augmentative and Alternative Communication. 1994, v. 10, n. 1, p. 27-59.

Submetido em 15 de agosto de 2013.

Aprovado em 04 de setembro de 2013.

Regina Yu Shon Chun: Docente do Curso de Graduação em Fonoaudiologia e do Programa de Mestrado Saúde, Interdisciplinaridade e Reabilitação da Faculdade de Ciências Médicas da Universidade Estadual de Campinas - UNICAMP, Campinas - São Paulo-SP - Brasil.

E-mail: reginayu@fcm.unicamp.br

Eliana Cristina Moreira: Fonoaudióloga e Mestre em Saúde da Criança e do Adolescente pela Faculdade de Ciências Médicas da Universidade Estadual de Campinas - UNICAMP, Campinas - São Paulo/SP - Brasil.

E-mail: elianacmoreira@gmail.com

Maria Tereza Maynard Santana: Fonoaudióloga e aluna do Programa de Mestrado Saúde, Interdisciplinaridade e Reabilitação da Faculdade de Ciências Médicas da Universidade Estadual de Campinas - UNICAMP, Campinas - São Paulo/SP - Brasil. E-mail: mariaterezamaynard@yahoo.com.br

Graziella Batista Dallaqua: Fonoaudióloga e aluna do Programa de Mestrado Saúde, Interdisciplinaridade e Reabilitação da Faculdade de Ciências Médicas da Universidade Estadual de Campinas - UNICAMP, Campinas - São Paulo/SP - Brasil.

E-mail: gradallaqua@yahoo.com.br 